\title{
Patienten-WC
}

\section{Mehr Aufmerksamkeit für das stille Örtchen}

\author{
Ansprechendes Design, leicht in der Pflege, sparsam im Verbrauch: \\ So wird das Patienten-WC in der Arztpraxis jedem Anspruch gerecht \\ und hinterlässt bei den Benutzern einen guten Eindruck.
}

S ie steht nicht im Rampenlicht wie die Empfangstheke, doch auch sie sollte in jedem Fall einen angenehmen Eindruck bei ihren Besuchern hinterlassen: die Patiententoilette.

\section{Vorausschauende Lageplanung}

Auf die Lage der Patiententoilette sollte in einer neuen Praxis unbedingt geachtet werden. Innenarchitekt Norbert Thöne aus Hamm, der sich auf Gesundheitsbau spezialisiert hat, rät: „Das WC sollte sich im vorderen Praxisbereich, nahe der Rezeption und des Wartezimmers befinden. So muss der Patient nicht lange nach der Toilette suchen, und die Mitarbeiter haben eine gewisse Kontrollmöglichkeit.“ Zudem werde der Praxisbetrieb r durch Patienten beeinträchtigt, die e zum Aufsuchen der Toilette immer b in den Behandlungsbereich laufen müssten.

Wird die Patiententoilette nachträglich verlegt, weil zum Beispiel ein Anschluss ans Labor entstehen soll, so erfordert das umfassende Eingriffe in die Haustechnik: Beund Entlüftungsleitungen müssen verlegt und neu installiert werden. Vor allem Abwasserleitungen können Probleme verursachen, da vielleicht am geplanten Standort keine

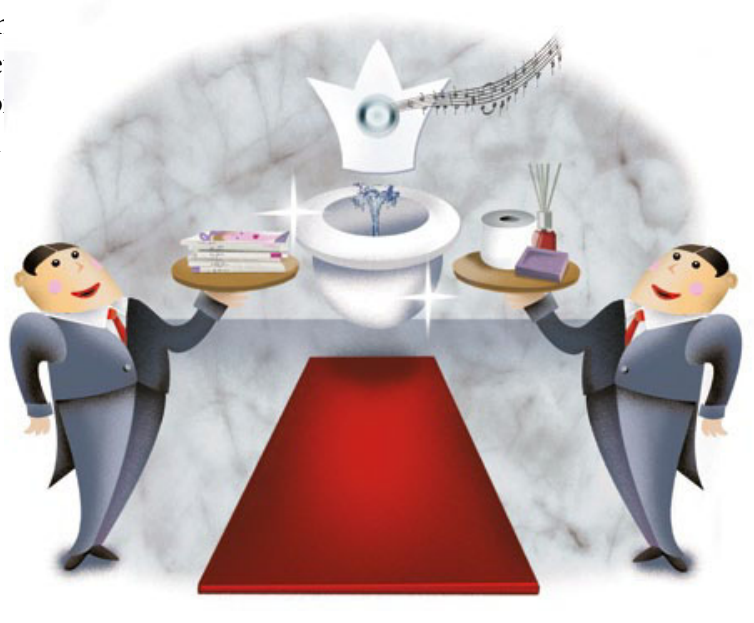
Fallrohre in der erforderlichen Größe vorhanden sind. „Grundsätzlich ist die Anbindung von Patienten-WC an das Labor sinnvoll und sollte Standard sein in Praxen, in denen Urinproben genommen werden", erklärt Thöne. Technisch sinnvoll sei die Zusammenlegung von allen in der Praxis notwendigen Sanitäranlagen, rät der Planer.

\section{Naturstein im Design}

Auch das Design der Sanitärräume kann so schön wie zweckmäßig sein. Innenarchitekt Thöne empfiehlt gerne Naturstein, aber auch andere Materialien: „Bei den Bodenbelägen kann man auch auf PVCBahnenware zurückgreifen. Die PVCBöden sollten allerdings verschweißt und fugenlos zehn Zentimeter an den Wänden hochgeführt werden." Ebenso viele Gestaltungsmöglichkeiten bieten Terrazzoböden. Dabei gilt immer: Die Böden müssen über die geforderte Rutschhemmung verfügen - in Arztpraxen ist die Klasse R10 vorgeschrieben. Bei den Armaturen rät Thöne auf Einhand- oder berührungslose Bedienung zu setzen. Die Armaturen sollten zudem über einen gesichert sein, um einen Verbrühschutz zu gewährleisten. Und: „Die Armatur sollte so gestaltet sein, dass die Bedienung keine Rätsel aufgibt!“ Temperaturbegrenzer thermostatisch ab-
Wer die vorgenannten Funktionen mit klassisch-zeitlosem Design kombinieren will, kann etwa auf das Angebot des dänischen Herstellers Vola zurückgreifen, das unverkennbar die Handschrift des Designers Arne Jacobsen trägt. „Ein Mediziner steht im Wettbewerb, er muss ein gewisses Ambiente schaffen. Und nicht selten schließen Patienten vom Ambiente auf die Leistung! Entscheidet sich der Arzt für klassische Elemente, setzt er damit ein Zeichen. Wer die Bayerische Staatssammlung mit ihrer Pinakothek besucht, kann Vola sowohl in der Sammlung, als auch in der Benutzung erleben“, sagt Daniela Darimont, Produktmanagerin bei Vola in München. Der Hersteller setzt seit jeher auf massiven Edelstahl und hat die Unterputzarmatur entwickelt.

\section{Moderner Gesundheitsbau}

Darimont empfiehlt für den Gesundheitsbau Unterputzarmaturen. „Sie bieten we:r Angriffsfläche! Das Beste ist, man cheidet sich für die elektronisch steubare, berührungslose Armatur mit iner vorgegebenen Nachlaufzeit, einer Hygienespülung, um stehendes Wasser in Rohrleitungen zu vermeiden." Die Durchflussmenge nahezu aller Vola-Waschtischarmaturen liegt bei fünf Litern pro Minute. Mithilfe von Durchflussmengenreglern lässt sich die Menge stufenweise auf 1,9 Litern reduzieren.

Auch bei den WC- und Urinalspülungen ist eine bedarfsgerechte, wassersparende Spülung mittlerweile Standard. „Dies kann durch eine elektronische Steuerung oder alternativ durch zwei unterschiedliche Spülmengen erfolgen. Oder über eine StartStopp-Funktion bei den Bedienelementen. Selbst ältere Urinale und WC lassen sich durch Elektroniumrüstsätze entsprechend nachrüsten", erklärt Architekt Thöne. 